\title{
A Experimentação na Educação em Química Verde: uma Análise de Propostas Didáticas Desenvolvidas por Licenciandos em Química de uma IES Federal Paulista
}

\author{
Experimentation in Green Chemistry Education: an Analysis of Teaching \\ Proposals Developed by Undergraduates of a Federal University in São Paulo
}

Rosivânia da Silva Andrade, (Dânia Gomes Zuin

\begin{abstract}
Palavras-chave Resumo Este trabalho busca analisar a inserção dos Princípios da Experimentação; Química Verde; Formação de Professores. Educação em Química Verde (EQV) nas propostas experimentais desenvolvidas pelos estudantes na disciplina de Experimentação na Educação Química do curso de Licenciatura em Química de uma Instituição de Ensino Superior (IES) federal localizada no estado de São Paulo. As propostas desenhadas pelos estudantes foram analisadas de acordo com os níveis de grau verde correspondentes aos Princípios da EQV. De acordo com as análises, verificou-se que as propostas caminharam de forma a contemplar níveis variáveis de grau verde, que, em geral, demonstraram um processo de aprendizagem em Química compatível à atuação responsável frente às dimensões tecnocientíficas, sociais, econômicas, geopolíticas e ambientais.
\end{abstract}

Keywords Experimentation; Green Chemistry; Teacher Education.

Abstract This paper analyzes the implementation of principles of Green Chemistry Education (GCE) in experimental proposals developed by students of the course "Experimentation in Chemical Education", from the teaching training program of a federal Higher Education Institution located in São Paulo, Brazil. The students' experiments were analyzed using the "levels of green" corresponding to the principles of the GCE. We verified that the experiments presented variable levels of green, which, in general, demonstrated a learning process in Chemistry that is compatible to the growing responsible action in technoscientific, social, economic, geopolitical and environmental dimensions. 


\section{Introdução}

As discussões acerca das preocupações ambientais tiveram início na década de 1940, mediante a Conferência Científica da Organização das Nações Unidas sobre Conservação e Utilização de Recursos em Lake Success, nos Estados Unidos. No entanto, o conceito de Química Verde (QV) surgiu apenas em 1998, definido por Paul Anastas e John Warner como uma abordagem para a criação, desenvolvimento e aplicação de produtos e de processos químicos que envolvam a redução ou a eliminação de substâncias nocivas à saúde humana ou ao ambiente (Anastas \& Warner, 1998; Lenardão et al., 2003).

Pensar a química de maneira inovadora e que beneficie a economia, as pessoas e o planeta implicam a necessidade de modelos de ensino que promovam um pensamento holístico, interdisciplinar e que aborde problemáticas e contextos reais. No entanto, ao analisar as produções de Química Verde na América Latina (Zuin et al., 2020) e, mais especificamente, no Brasil (Zuin \& Marques, 2015), percebe-se a ausência de trabalhos que insiram problemas sociocientíficos, os quais direcionam a aprendizagem para a tomada de consciência da construção dinâmica do conhecimento e do reconhecimento de suas limitações para uma aplicação melhor e mais útil.

A introdução dos princípios e da filosofia da Química Verde nas disciplinas de Química, seja na Educação em nível básico ou superior, ao utilizar questões controversas, constitui um dos pilares centrais para a construção de uma abordagem química contemporânea e mais ampla (Zuin, 2011; Zuin \& Pacca, 2012; Zuin et al., 2019).

Ao discutir a inserção da QV na formação inicial de professores de Química, Zuin (2013) propõe que o desenvolvimento de práticas experimentais verdes não se reduza apenas à substituição de reagentes menos perigosos, por exemplo, mas que inclua problemáticas socioambientais. Para que essa inclusão ocorra, a abordagem da Química Verde na formação de professores deve ser entendida por meio de seus princípios técnicos de forma mais ampla, para que possa enfrentar as complexas relações quando pensamos em ambiente, sociedade, educação e modos de produção e reprodução da vida.

Uma maneira de incorporar a Química Verde aos processos de formação é incentivar o uso de atividades laboratoriais que permitam aos estudantes refletir acerca do design do experimento - simplicidade, recuperação e reutilização de resíduos, prevenção de acidentes, redução de matéria-prima na fonte - ao mesmo tempo que integrem questões sociocientíficas e possibilitem a formação de professores com uma visão educacional e responsável da Química (Kirchhoff, 2001; Montes et al., 2006; Kitchens et al., 2006; Eilks et al., 2017).

Por conseguinte, é necessário propiciar aos estudantes práticas e instrumentos de formação que favoreçam a problematização no desenvolvimento de atividades experimentais verdes (Zuin, 2013; Zandonai et al., 2014; Almeida \& Biajone, 2007; Gonçalves \& Marques, 2016). Essa necessidade também é evidenciada no Projeto Pedagógico do Curso de Licenciatura em Química, que propõe que o licenciando deva ser desafiado a exercitar sua criatividade na resolução de problemas socioambientais. 
Contudo, o que se observa é um trabalho puramente mecânico de execução de um roteiro sistematizado que se pauta na (re)produção de resultados efêmeros, sendo, portanto, necessário o uso de instrumentos que excedam os fundamentos técnicos da Química Verde, restritos aos seus princípios, à análise do processo e ao desempenho do material (Andraos \& Sayed, 2007; Morales Galicia, 2011; Ribeiro \& Machado, 2013; VargasRodríguez, 2016), inserindo aspectos educacionais inerentes à formação docente.

Tendo em vista a necessidade de uma transição de práticas experimentais para além da aprendizagem técnica, esta pesquisa pretende responder à seguinte questão: $\mathrm{o}$ trabalho experimental nos laboratórios de ensino está sendo desenvolvido em direção aos desafios interligados à inserção da Química Verde, especificamente em uma disciplina de experimentação de um curso de licenciatura de Química de uma IES pública do Estado de SP?

Nesse sentido, a pesquisa analisa as práticas experimentais desenvolvidas pelos estudantes a partir dos Princípios da Educação em Química Verde (EQV). No próximo tópico são apresentados os Princípios da EQV, os quais fundamentam a pesquisa.

\section{Os Princípios da Educação em Química Verde}

Os Princípios da Educação em Química Verde compreendem um novo olhar para auxiliar a introdução da Química Verde na Educação Química (Saqueto, 2015). São eles:

1. Problematização/Contextualização. Introdução de temas cotidianos socioambientais e econômicos nas práticas experimentais por meio da problematização, contextualização e abordagem CTS (Ciência, Tecnologia e Sociedade), ou seja, por meio da criação de situações-problema para iniciar aulas experimentais.

2. Incentivo à pesquisa. Desenvolvimento de atitudes investigativas, da autonomia e da leitura crítica da sociedade para a tomada de decisões especialmente aquelas ambientalmente conscientes - para a produção de novos processos, novas tecnologias e de substâncias que sejam verdes em sua concepção.

3. Atenção aos produtos e processos. Ressignificação dos sentidos de prevenção e minimização da geração de produtos principais, coprodutos e resíduos, em que a não geração de resíduo deve ser priorizada.

4. Redução da geração e do uso de quaisquer materiais perigosos. Inserção do estudante no processo de avaliação de risco, para que possa minimizar o potencial para acidentes químicos, escolhendo experimentos que utilizem e gerem substâncias não perigosas, com pouca ou nenhuma toxicidade à saúde humana e ao ambiente, mobilizando os conteúdos conceituais e procedimentais a serem aprendidos. 
5. Preferência à utilização de reagentes de fontes renováveis, de fácil degradação e que sejam obtidos no entorno da situação de ensino. Evitar o uso de reagentes de fontes não renováveis, utilizando reagentes disponíveis no meio ambiente, por serem de fácil acesso e aproximarem-se ao contexto em que os estudantes estão inseridos, além de produzirem, em sua maioria, resíduos biodegradáveis.

6. Redução do consumo de energia elétrica e de água. Os experimentos químicos, sempre que possível, devem ser conduzidos a temperatura e pressão ambientes e ter uma utilização ínfima de água, de forma a minimizar o desperdício e manter o funcionamento adequado do experimento.

Os Princípios da Educação em Química Verde, alinhados às Diretrizes Curriculares Nacionais para os Cursos de Química (CNE/CES, 2001), ressaltam a importância de se abordar conteúdos atitudinais no ensino, além dos conceituais e procedimentais. Dentre os seis princípios descritos, os princípios "Problematização/Contextualização" e "Incentivo à pesquisa" podem ser considerados essenciais para o rompimento da aplicação exclusivamente técnica da Química Verde.

A aplicação destes princípios, em um movimento de complementariedade, apresenta-se como uma via de reflexão crítica e de desenvolvimento da autonomia durante o processo de aprendizagem - elementos fundamentais para a formação docente - além da consciência socioambiental indispensável para a atuação dos alunos como indivíduos em sociedade.

Portanto, os princípios para a Educação em Química Verde descritos por Saqueto (2015) contribuem para que os estudantes adquiram habilidades e competências para além do conhecimento teórico, desenvolvendo valores e responsabilidades que possibilitem uma futura atuação profissional competente e compromissada com os critérios humanísticos, éticos, legais e de rigor científico, os quais, de acordo com as Diretrizes Curriculares Nacionais para os Cursos de Química (CNE/CES, 2001), são essenciais para sua formação.

Os alcances da EQV em experimentos didáticos podem ser avaliados por diferentes métricas. Adotou-se nesse trabalho a Estrela Verde da Educação (EQV), que se baseia na EV (Ribeiro et al., 2010) e nos princípios da EQV. Para cada princípio o grau verde pode variar de 1 a 3 , de forma crescente, conforme sua aderência ao princípio (Ribeiro et al., 2010b; Saqueto, 2015). Os seis princípios são descritos na Figura 1. 
Figura 1. Princípios da Educação em Química Verde e escalas do grau verde para construir a EV

\begin{tabular}{|c|c|c|c|c|}
\hline & GRAU VERDE 1 & GRAU VERDE 2 & GRAU VERDE 3 \\
\hline \multirow{6}{*}{ 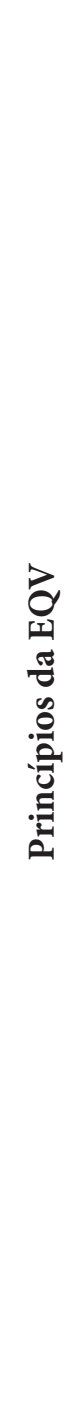 } & \multirow[b]{2}{*}{ P1 } & \multicolumn{3}{|c|}{ Problematização/Contextualização } \\
\hline & & $\begin{array}{l}\text { a) Experimento sem uso } \\
\text { de Problematização/ } \\
\text { Contextualização e } \\
\text { abordagem CTS }\end{array}$ & $\begin{array}{l}\text { a) Inclui contextualização } \\
\text { sem problematização; ou } \\
\text { b) Utiliza como reagentes } \\
\text { produtos presentes no } \\
\text { cotidiano dos estudantes }\end{array}$ & $\begin{array}{l}\text { a) Inclui problematização } \\
\text { e contextualização com } \\
\text { abordagem CTS }\end{array}$ \\
\hline & & \multicolumn{3}{|c|}{ Incentivo à pesquisa } \\
\hline & $\mathbf{P 2}$ & $\begin{array}{l}\text { a) Não incentiva a pesquisa; } \\
\text { experimentos somente } \\
\text { reprodutivos }\end{array}$ & $\begin{array}{l}\text { a) Além do relatório, o } \\
\text { estudante pode apresentar } \\
\text { seu experimento } \\
\text { (seminário) com reflexão de } \\
\text { sua prática; e } \\
\text { b) Possibilita ao estudante } \\
\text { tomar decisões durante o } \\
\text { experimento (roteiro com } \\
\text { abertura) }\end{array}$ & $\begin{array}{l}\text { a) Incentiva a } \\
\text { pesquisa por meio de } \\
\text { experimentos livres }\end{array}$ \\
\hline & & \multicolumn{3}{|c|}{ Atenção aos produtos e processos } \\
\hline & $\mathbf{P 3}$ & $\begin{array}{l}\text { a) Sem qualquer forma de } \\
\text { minimização de resíduos }\end{array}$ & $\begin{array}{l}\text { a) Não gera resíduo } \\
\text { (economia de átomos); ou } \\
\text { b)Prática que minimiza } \\
\text { resíduo (quantidade, } \\
\text { concentração, tratamento, } \\
\text { reutilização do produto } \\
\text { gerado) }\end{array}$ & $\begin{array}{l}\text { a) Não gera produto/ } \\
\text { resíduo (utiliza } \\
\text { vídeo, demonstração, } \\
\text { simulação); ou } \\
\text { b) Introdução que faz } \\
\text { repensar a prática para } \\
\text { evitar a geração de } \\
\text { resíduos; ou } \\
\text { c) Total } \\
\text { reaproveitamento dos } \\
\text { resíduos }\end{array}$ \\
\hline
\end{tabular}

Fonte: adaptado de Saqueto, 2015. 
Figura 1. Princípios da Educação em Química Verde e escalas do grau verde para construir a EV (continuação)



Fonte: adaptado de Saqueto, 2015. 
Figura 1. Princípios da Educação em Química Verde e escalas do grau verde para construir a EV (continuação)

\begin{tabular}{|c|c|c|c|c|}
\hline & GRAU VERDE 1 & GRAU VERDE 2 & GRAU VERDE 3 \\
\hline \multirow{3}{*}{ 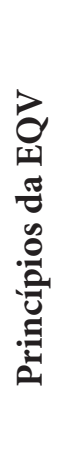 } & \multirow{3}{*}{ P6 } & \multicolumn{3}{|c|}{ Redução do consumo de energia elétrica e de água } \\
\hline & & $\begin{array}{l}\text { a) Utiliza aparelhos que } \\
\text { consomem mais energia na } \\
\text { relação tempo/consumo; } \\
\text { ou }\end{array}$ & $\begin{array}{l}\text { a) Utiliza aparelhos que } \\
\text { consomem menos energia } \\
\text { na relação tempo/consumo, } \\
\text { como micro-ondas; ou }\end{array}$ & $\begin{array}{l}\text { a) Não consome energia } \\
\text { elétrica (exemplo: sem } \\
\text { aquecimento/agitação); } \\
\text { e }\end{array}$ \\
\hline & & $\begin{array}{l}\text { b) Alto tempo de consumo; } \\
\text { ou } \\
\text { c) Consumo desnecessário } \\
\text { de água }\end{array}$ & $\begin{array}{l}\text { b) Baixo tempo de consumo } \\
\text { de energia elétrica; ou } \\
\text { c) Utiliza fontes de energia } \\
\text { alternativas }\end{array}$ & b) Não desperdiça água \\
\hline
\end{tabular}

Fonte: adaptado de Saqueto, 2015.

Para uma visualização qualitativa do grau verde de um experimento didático, de modo a permitir a comparação de duas ou mais práticas, a Estrela Verde da Educação (EVE) é estabelecida a partir da soma dos valores atribuídos a cada princípio da Educação em Química Verde, sendo 3 o valor máximo para cada extremidade das pontas (Figura 2 , na qual todos os princípios apresentam aspectos que os categorizam com grau verde 3) e 1 o valor mínimo para cada extremidade das pontas (Figura 3, na qual todos os princípios apresentam aspectos que os categorizam com grau verde 1).

Figura 2. Estrela verde da Educação (EVE) de maior grau verde possível

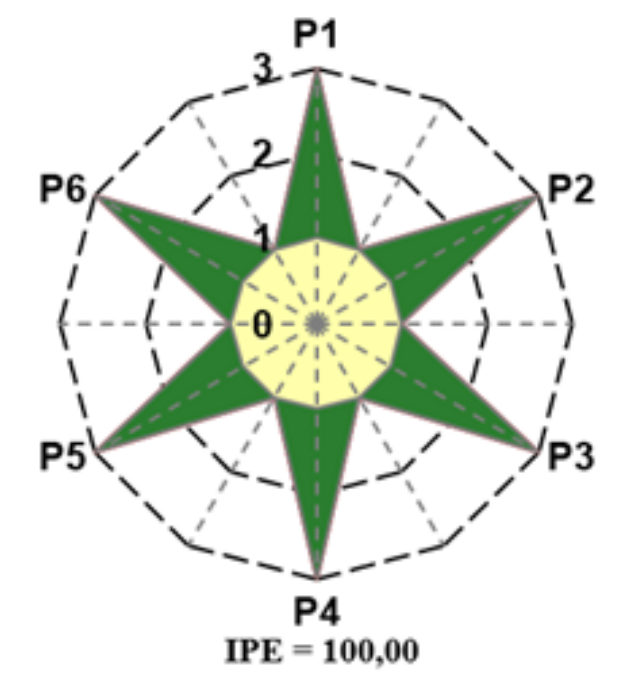

Fonte: adaptado de Ribeiro et al., 2010b. 
Figura 3. Estrela verde da Educação (EVE) de menor grau verde possível

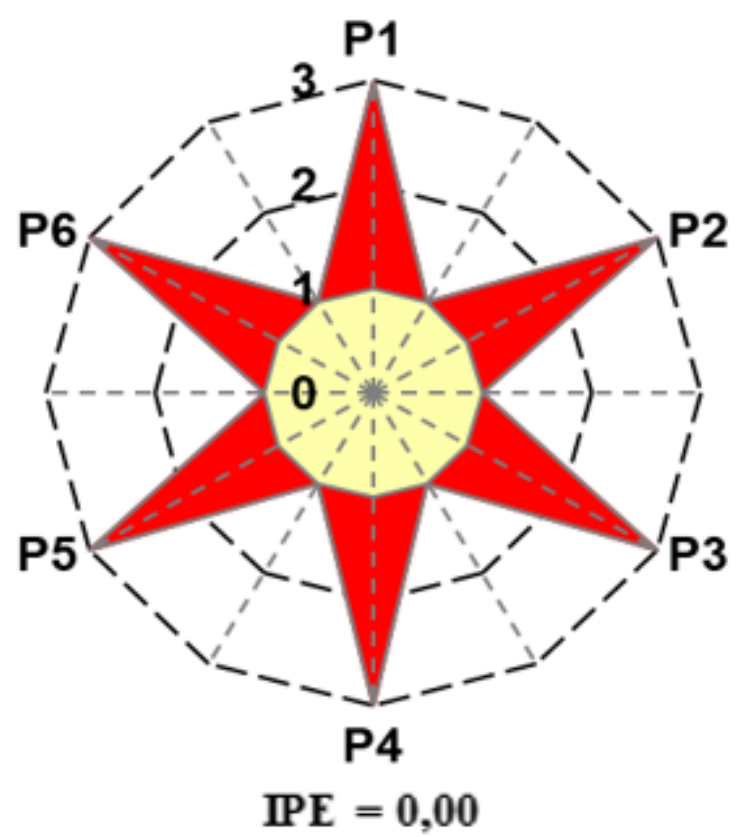

Fonte: adaptado de Ribeiro et al., 2010b.

Os Princípios da Educação em Química Verde são desenhados para que os professores, estudantes e demais agentes educativos possam avaliar os alcances e limites de práticas experimentais de forma crítica, considerando as relações científicas, tecnológicas, sociais e ambientais.

\section{Metodologia}

Nesta pesquisa foi adotada a abordagem qualitativa como metodologia de investigação, por permitir um trabalho direto no campo e fornecer material de pesquisa, como também por apresentar especial relevância para a pesquisa contemporânea na área de Educação (Flick, 2009). A pesquisa ocorreu na disciplina de Experimentação na Educação Química do curso de Licenciatura em Química de uma IES federal paulista. Os sujeitos envolvidos foram estudantes da respectiva disciplina. Vale ressaltar que, dos 20 estudantes, 16 deles já haviam cursado a disciplina de Química Verde - também presente na matriz curricular do referido curso - , possibilitando uma integração de conceitos para a elaboração das propostas experimentais. Todos foram convidados e aceitaram participar do estudo, assinando o Termo de Consentimento Livre e Esclarecido (TCLE), conforme regulamentado pelo Comitê de Ética em Pesquisa.

O principal objeto de análise deste estudo foram as propostas experimentais elaboradas pelos estudantes, mas também foi empregado o método da observação (Creswell, 2010), no qual um bloco de anotações foi utilizado para tomar notas descritivas e reflexivas, visando uma melhor compreensão da construção das propostas experimentais. A investigação foi organizada em seis momentos: iniciou-se a partir de 
discussões com os estudantes sobre a utilização de laboratórios no ensino de Química e sobre como organizar e conduzir práticas que possibilitem o desenvolvimento de experiências formativas verdes (Silva, Machado e Tunes 2010; Zandonai et al., 2014; Zuin \& Zuin 2017).

No segundo momento, ocorreu a seleção dos temas e dos experimentos que seriam integrados ao tema escolhido, destacando que os grupos poderiam escolher temas iguais, mas que os experimentos deveriam, necessariamente, ser diferentes. Cada grupo selecionou seu tema de interesse a partir de uma lista indicada pelos professores das turmas da Educação Básica, tendo como referência a Proposta Curricular do Estado de São Paulo e os livros didáticos ${ }^{1}$ utilizados na escola, enquanto a escolha dos experimentos foi livre, de acordo com o tema escolhido. Em seguida, no terceiro momento, foi organizada uma roda de discussões com todos os grupos para apresentação e análise de suas escolhas. No quarto momento, ocorreram os testes dos experimentos.

No quinto momento, houve a aplicação das propostas experimentais para estudantes da Educação Básica e, por fim, no sexto momento, a redação de relatórios e a apresentação dos resultados referentes à aplicação dos experimentos a todos os licenciandos, professores, monitores e técnico de laboratório, promovendo discussões e reflexão crítica e originando a versão final das propostas experimentais.

Vale ressaltar que cada grupo escolheu dois experimentos, totalizando 14 propostas experimentais integradas aos temas: combustíveis, estequiometria, eletroquímica, concentração, solubilidade, atomística, consumo e produção de resíduos sólidos, distribuídos de acordo com o Figura 4.

Figura 4. Distribuição dos temas por Grupo

\begin{tabular}{|c|l|l|l|}
\hline Grupos & Temas & Experimentos & Descrição ${ }^{2}$ \\
\hline \multirow{2}{*}{ G1 } & Combustíveis & $\begin{array}{l}\text { Adulteração de } \\
\text { combustíveis }\end{array}$ & $\begin{array}{l}\text { Reconhecer amostras de gasolina fora } \\
\text { do padrão estabelecido pela Agência } \\
\text { Nacional de Petróleo, Gás Natural e } \\
\text { Biocombustíveis (ANP). }\end{array}$ \\
\hline & \multirow{2}{*}{ G2 } & $\begin{array}{l}\text { Alimentos: os } \\
\text { combustíveis do nosso } \\
\text { corpo }\end{array}$ & $\begin{array}{l}\text { Identificar como os alimentos } \\
\text { funcionam como fonte de energia para o } \\
\text { organismo. }\end{array}$ \\
\cline { 3 - 4 } & Um bafômetro caseiro & $\begin{array}{l}\text { Abordar os conceitos estequiométricos } \\
\text { envolvidos na reação de detecção do } \\
\text { álcool, assim como problematizar } \\
\text { os aspectos sociais envolvendo os } \\
\text { problemas relacionados ao alcoolismo. }\end{array}$ \\
\cline { 3 - 4 } & $\begin{array}{l}\text { Análise de uma cena de } \\
\text { crime fictícia }\end{array}$ & $\begin{array}{l}\text { Reconhecer o papel da Química Forense } \\
\text { e suas aplicações a partir da reação de } \\
\text { quimiluminescência. }\end{array}$ \\
\hline
\end{tabular}

Fonte: elaboração própria.

1 Coleção de Química da autora Martha Reis PNLD (2014-2017).

2 As descrições das propostas experimentais foram elaboradas pelos grupos correspondentes. 
Figura 4. Distribuição dos temas por Grupo (continuação)

\begin{tabular}{|c|c|c|c|}
\hline Grupos & Temas & Experimentos & Descrição \\
\hline \multirow[b]{2}{*}{ G3 } & \multirow[t]{2}{*}{ Eletroquímica } & Pilha molhada & $\begin{array}{l}\text { Analisar o funcionamento de uma célula } \\
\text { eletroquímica e identificar suas relações } \\
\text { cotidianas. }\end{array}$ \\
\hline & & Descolorindo a água & $\begin{array}{l}\text { Demonstrar os diferentes potenciais de } \\
\text { redução entre os átomos e as trocas de } \\
\text { íons que ocorrem em uma solução de } \\
\text { diferentes metais. }\end{array}$ \\
\hline \multirow{2}{*}{ G4 } & \multirow[t]{2}{*}{ Concentração } & $\begin{array}{l}\text { MasterQuimJuice: O } \\
\text { jurado é você }\end{array}$ & $\begin{array}{l}\text { Demonstrar e avaliar a qualidade das } \\
\text { bebidas a partir dos compostos presentes } \\
\text { nas frutas. }\end{array}$ \\
\hline & & $\begin{array}{l}\text { Determinação da } \\
\text { Vitamina C em frutas }\end{array}$ & $\begin{array}{l}\text { Determinar a quantidade de vitamina } \\
\mathrm{C} \text { em sucos de frutas industriais e } \\
\text { caseiros. }\end{array}$ \\
\hline \multirow[t]{2}{*}{ G5 } & \multirow[t]{2}{*}{ Solubilidade } & A torre de acetato & $\begin{array}{l}\text { Abordar os conceitos de solução } \\
\text { supersaturada, precipitado e } \\
\text { sobrenadante. }\end{array}$ \\
\hline & & $\begin{array}{l}\text { Soprando na água de } \\
\text { cal }\end{array}$ & $\begin{array}{l}\text { Abordar conceitos sobre perturbação de } \\
\text { equilíbrio e o Princípio de Le Chatelier. }\end{array}$ \\
\hline \multirow[t]{2}{*}{ G6 } & \multirow[t]{2}{*}{ Atomística } & Caixa preta & $\begin{array}{l}\text { Aproximar os estudantes da realidade } \\
\text { científica da química, compreendendo } \\
\text { seu desenvolvimento a partir da } \\
\text { observação indireta de fenômenos, de } \\
\text { evidências, da formulação de hipóteses } \\
\text { e refletindo sobre a diferença entre o } \\
\text { modelo científico e o senso comum. }\end{array}$ \\
\hline & & Teste de chama & $\begin{array}{l}\text { Observar as diferenças de cores nas } \\
\text { chamas formadas a partir de soluções } \\
\text { com cátions distintos. }\end{array}$ \\
\hline \multirow[b]{2}{*}{ G7 } & \multirow[t]{2}{*}{$\begin{array}{l}\text { Consumo e } \\
\text { produção de resíduos } \\
\text { sólidos }\end{array}$} & $\begin{array}{l}\text { Reciclagem de papel } \\
\text { para embalagens, que } \\
\text { tal? }\end{array}$ & $\begin{array}{l}\text { Demonstrar a reciclagem de papel } \\
\text { em pequena escala e a aplicação desse } \\
\text { material em embalagens. }\end{array}$ \\
\hline & & $\begin{array}{l}\text { Uma alternativa } \\
\text { perfumada }\end{array}$ & $\begin{array}{l}\text { Proporcionar a aprendizagem de } \\
\text { técnicas de produção de sabonete } \\
\text { artesanal aplicando conceitos } \\
\text { de Química Orgânica de forma } \\
\text { alternativa. }\end{array}$ \\
\hline
\end{tabular}

Fonte: elaboração própria.

As propostas experimentais, em sua versão final, foram analisadas a partir dos critérios estabelecidos para cada princípio da Educação em Química Verde (Quadro 1), identificando seus níveis de grau verde correspondentes. Também foi empregada a métrica Estrela Verde da Educação (EVE), utilizando as escalas do grau verde para cada princípio da EQV a fim de verificar as variáveis entre as práticas comumente aplicadas 
e as novas orientações atribuídas ao experimento. Para isso, foram selecionados os experimentos "Um bafômetro caseiro" e "Pilha molhada", por abordarem conceitos químicos difíceis de serem compreendidos (Furió \& Furió, 2000; Cárdenas, 2006; Pozo \& Crespo, 2009), assim como por serem recorrentes em aplicações didáticas das temáticas estequiometria e eletroquímica (Santos et al., 2014; Ferreira et al., 1997; Rubinger \& Braathen, 2012; Guimarães \& Castro, 2018; Cruz \& Filho, 2004; Rodrigues et al., 2016; Barreto et al., 2017).

\section{Resultados e discussões}

$\mathrm{Na}$ análise do grau verde para os experimentos elaborados pelos grupos foi verificado que a maioria das propostas experimentais (nove) apresentou grau verde G2 para o Princípio P1, por interligar os conceitos científicos que fundamentam o experimento e questões sociais, ambientais e tecnológicas por meio da contextualização. Além disso, os estudantes utilizaram como reagentes produtos presentes no cotidiano e de fácil acesso.

Para Bazzo (2002), esses aspectos possibilitam o desenvolvimento da capacidade crítica e reflexiva dos estudantes sobre assuntos que integram sua vida como indivíduos e como sociedade. Assim, os estudantes puderam compreender que o conhecimento químico não é útil apenas para o trabalho no laboratório, para a pesquisa ou descobertas científicas, mas para a vida cotidiana e profissional (Pozo \& Crespo, 2009; Zuin et al., 2019).

Ainda para o Princípio P1, três experimentos apresentaram grau verde G1 por não incluírem aspectos sociais, tecnológicos ou ambientais em sua abordagem, restringindose apenas à compreensão conceitual. Desse modo, a forma como os experimentos foram organizados não contribuem em grande extensão para o desenvolvimento de atitudes e valores, que, para Santos \& Mortimer (2002) e Zuin \& Zuin (2017), são indispensáveis para a formação de um cidadão crítico.

No entanto, houve duas propostas experimentais que atingiram o grau verde máximo G3, por oferecerem uma problematização sobre o tema abordado, apresentando não apenas o papel da ciência na resolução da situação-problema, mas os aspectos sociais e ambientais envolvidos. Incluir a problematização como propulsora no processo de aprendizagem implica o desenvolvimento da consciência crítica. A atividade experimental, nesse contexto, possibilitou, em concordância com Delizoicov et al., (2002), o desenvolvimento de processos cognitivos de análise e interpretação do conhecimento incorporado, promovendo inter-relações com questões sociais, tecnológicas e ambientais.

Em relação ao Princípio P2, onze das quatorze propostas obtiveram grau verde G2, em função dos experimentos variarem no grau de abertura entre nível entreaberto e ligeiramente aberto (Jiménez Valverde et al., 2006). Esses níveis de abertura requerem do estudante processos cognitivos de compreensão e de aplicação do conhecimento, permitindo tomada de decisão quanto à resolução da situação-problema apresentada como ponto inicial do experimento e quanto às conclusões. 
Utilizar práticas experimentais que requerem a tomada de decisão quanto aos processos e soluções contribui para que os alunos desenvolvam dimensões importantes em sua formação, como "o aprendizado de uma convivência cooperativa com os colegas, respeito às diferentes formas de pensar, o cuidado na avaliação de uma afirmação e autoconfiança para defesa de pontos de vista" (Capecchi, 2013, p. 37).

Em contrapartida, três experimentos receberam grau G1 para esse princípio, pois possuíam nível de abertura fechado. Esse nível requer dos estudantes apenas processos cognitivos de conhecimento, permitindo somente elaborar conclusões acerca de questões conceituais inicialmente abordadas ao realizar o experimento.

No Princípio P3, a maioria das propostas (onze) alcançou grau verde G3, por escolherem e/ou redesenharem, quando necessário, práticas experimentais que evitassem a geração de resíduos. Como exemplo podemos citar o Grupo 2, que realizou diferentes testes de concentração e de quantidade dos reagentes para que houvesse uma minimização/prevenção da geração de coprodutos e de resíduos. As demais propostas (três) obtiveram grau verde G2.

Desenhar o processo de uma atividade experimental tendo em vista a minimização dos impactos ambientais fornece aos estudantes um pensamento crítico sobre como melhor conceber produtos e processos que sejam economicamente viáveis e ambientalmente seguros. O alcance desse grau verde, de acordo com Machado (2011), indica que esses estudantes estão aptos para desenvolver um design para o ambiente (DfE, Design for Environment) na concepção de produtos e processos que tenham medidas proativas que reduzam ou eliminem eventuais efeitos negativos no ambiente.

Todas as propostas experimentais foram desenhadas de modo a evitar a geração e o uso de quaisquer materiais perigosos, obtendo grau verde G3 para o Princípio P4, por utilizarem substâncias inócuas ou adaptarem as concentrações das substâncias para que houvesse uma economia de átomos, o que permitiu que a geração de resíduos fosse muito baixa - não causando riscos à saúde nem ao ambiente.

Essa preocupação quanto às substâncias utilizadas nos processos químicos é enfatizada por Saqueto (2015), principalmente em relação ao ensino, para que os experimentos não gerem substâncias perigosas à saúde humana e ao ambiente, de acordo com os conteúdos conceituais e procedimentais que envolvem a prática.

Em relação ao Princípio P5, referente aos tipos de reagentes empregados nas propostas experimentais, a maioria das propostas (oito) apresentou grau verde G2, em função da escolha de reagentes convencionais que reduzem o custo ambiental e econômico dos produtos ou por utilizarem reagentes de fontes renováveis e de fácil degradação.

As seis demais propostas obtiveram grau verde G3, por utilizarem reagentes naturais. No caso do Grupo 1 houve o uso de alimentos como castanha e amendoim e, para o Grupo 4, diferentes frutas, como laranja, abacaxi, morango e limão. A utilização de materiais naturais, derivados de plantas, de outras fontes biológicas renováveis ou reciclados também é apresentada por Lenardão et al., (2003) como uma necessidade para a inserção da Química Verde nas práticas laboratoriais. 
Nove propostas alcançaram grau verde G3 quanto ao Princípio P6, por não consumirem energia elétrica e não desperdiçarem (ou por reutilizarem) água durante o processo experimental. Já as demais propostas (cinco) optaram por utilizar baixo consumo de energia elétrica e, por esta razão, mostraram grau verde G2.

$\mathrm{Na}$ Figura 5 é possível verificar a distribuição do grau verde de cada princípio de acordo com os grupos, assim como a frequência com que os graus aparecerem nas propostas apresentadas:

Figura 5. Distribuição do grau verde das propostas experimentais por grupo

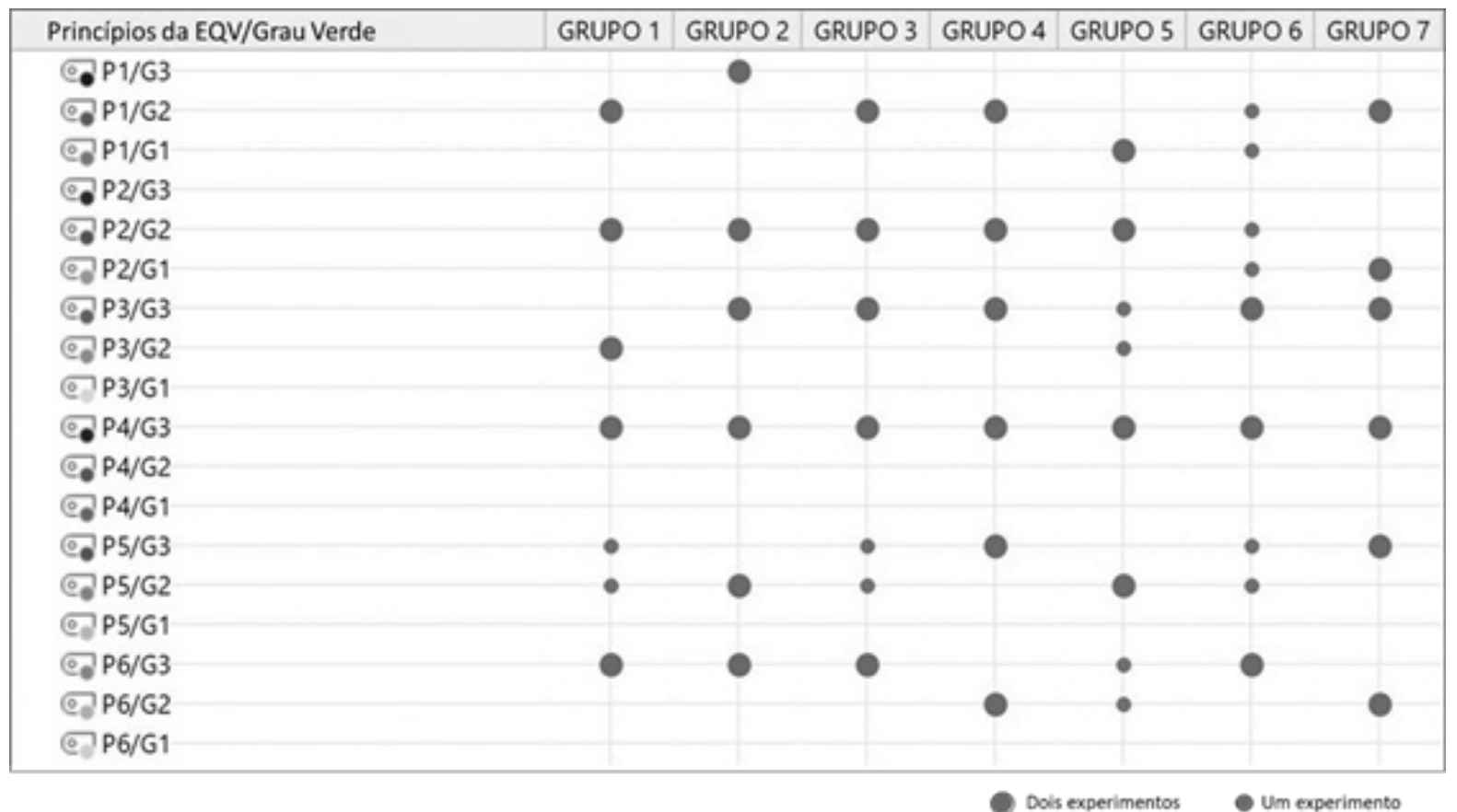

Fonte: elaboração própria.

De acordo com a Figura 5, as propostas experimentais caminharam de forma a contemplar níveis de grau verde que mostram processos de aprendizagem em Química adequados a uma atuação responsável frente aos aspectos sociais e ambientais que estão presentes nos experimentos. Portanto, os conteúdos de Química associados aos demais conhecimentos pedagógicos obtidos durante a disciplina mostraram um processo educativo capaz de proporcionar a alfabetização e literacia científica e pedagógica, como objetivado.

Para compreender as variáveis entre as práticas comumente aplicadas e as novas orientações atribuídas ao experimento a partir da Química Verde é apresentado um contraste entre as propostas didáticas tradicionais ${ }^{3} \mathrm{e}$ as propostas didáticas $\mathrm{EQV}^{4}$ sobre

3 Propostas didáticas tradicionais: são aquelas que estão centradas apenas na execução do experimento; se constituem somente de uma ligeira introdução com o objetivo do experimento, os materiais, reagentes e procedimentos.

4 Propostas didáticas EQV: são aquelas que estão centradas na formação crítica e socioambiental; incorporam, além dos itens básicos de materiais, reagentes e procedimentos, a filosofia e os princípios da Química Verde, a problematização e/ou contextualização do conceito e incentivam a pesquisa a partir de diferentes níveis de abertura. 
os temas estequiometria e eletroquímica. Para tanto, foi utilizada a métrica Estrela Verde da Educação (EVE), construída a partir da identificação do grau verde de cada proposta para cada princípio da EQV.

Partindo do tema estequiometria, o Grupo G2 apresentou como uma de suas propostas a construção de um bafômetro com o objetivo de abordar os conceitos estequiométricos envolvidos na reação de detecção do álcool, assim como problematizar os aspectos sociais relacionados ao alcoolismo. Tradicionalmente, a aplicação desse experimento tem sido unicamente ilustrativa, de modo a contemplar apenas os conceitos científicos e o processo de reação (Ferreira et al., 1997; Rubinger \& Braathen, 2012; Santos et al., 2014), o que não favorece a formação crítica dos estudantes considerando, por exemplo, a grande porcentagem de acidentes de trânsito causados por pessoas alcoolizadas.

Tomando as distinções entre a nova abordagem experimental (pautada nos princípios da EQV) e a abordagem tradicional desse experimento, são apresentadas as Estrelas Verdes da Educação (EVE) e seus respectivos índices de preenchimento para ambas as abordagens (Figura 6):

Figura 6. EVE de um experimento de bafômetro seguindo os princípios da EQV em comparação à proposta didática tradicional

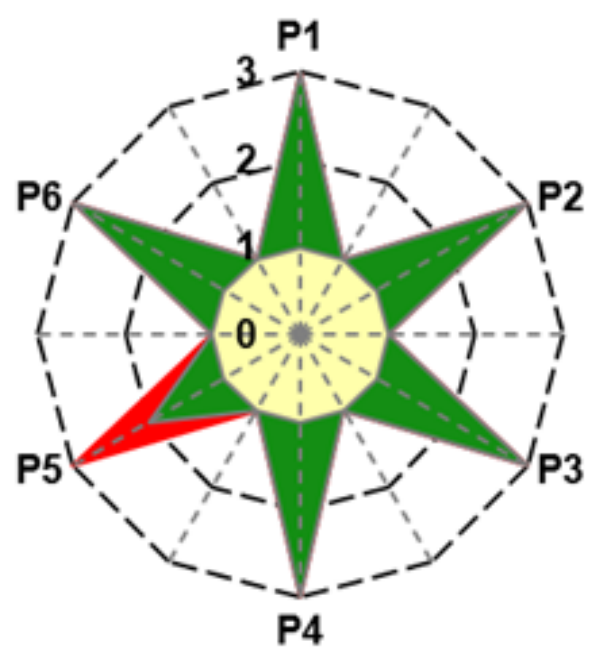

IPE $=91,67$

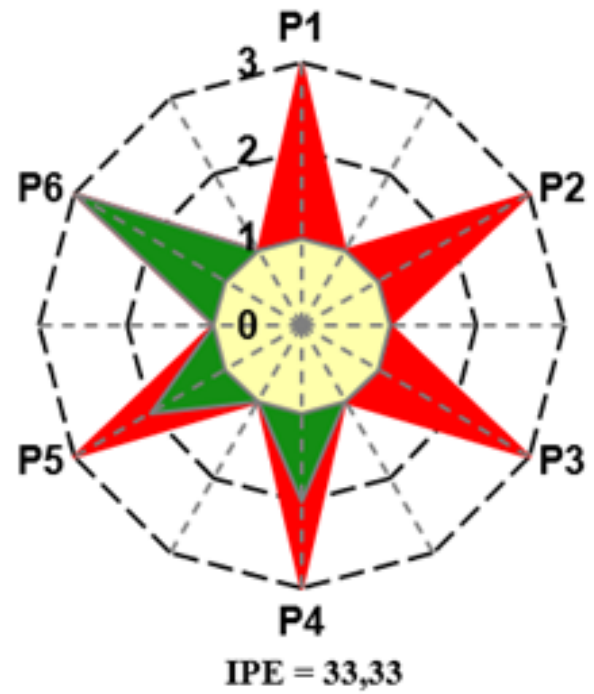

Bafömetro Tradicional

Fonte: elaboração própria.

Ao analisar ambas as figuras e seus respectivos índices de preenchimento da Estrela Verde da Educação (EVE), é observado que no Princípio P1 a proposta desenhada pelos estudantes recebeu grau verde G3, enquanto a proposta tradicional recebeu grau verde G1, por não incluir a problematização como propulsora da atividade experimental. 
No Princípio P2, a proposta didática EQV mostrou grau verde G3, por apresentar um grau de abertura que permite um processo cognitivo de elevada ordem e a aplicação dos conceitos abordados. Por outro lado, a proposta didática tradicional tem sido desenvolvida com uma abordagem demonstrativa, sem nenhuma abertura para investigação - aspectos que caracterizam o grau verde G1. Em relação ao Princípio P3, a proposta desenhada pelos estudantes recebeu grau verde G3, pois foram abordadas as características para se evitar a geração de resíduos. Para chegar a esse grau verde, o Grupo 2 realizou diferentes testes de concentração e de quantidade dos reagentes, alcançando uma economia de átomos e conservando os aspectos reacionais do experimento, enquanto nos modelos tradicionais desse experimento nenhuma forma de minimização de resíduos é descrita, indicando grau verde G1.

No Princípio $\mathrm{P} 4$, ambas as propostas utilizaram substâncias de risco moderado à saúde e ao ambiente, como ácido sulfúrico e dicromato de potássio. No entanto, na proposta dos alunos, as concentrações e quantidades utilizadas dessas substâncias foram reduzidas, uma vez que houve uma adaptação para uma economia de átomos, o que caracteriza um grau verde G3 para essa proposta, enquanto a proposta tradicional obteve grau verde G2.

Quanto ao Princípio P5, ambas as propostas apresentaram grau verde G2, por não utilizarem reagentes naturais e de fácil degradação, mas empregarem materiais nacionais, o que reduz o custo ambiental e econômico dos produtos. O Princípio P6 foi identificado com grau verde G3 também para ambos os experimentos, dado que eles não consumiram energia elétrica e houve uso racional de água em seus procedimentos.

Observando o grau verde para cada proposta experimental, verificou-se que a proposta desenhada pelos licenciandos - bem como a EVE correspondente ofereceu ferramentas e habilidades para que os alunos pudessem tomar decisões de forma consciente sobre o tema a ser abordado, apresentando atividades com viés dialógico e problematizador. Esse modelo, ao contrário dos experimentos puramente demonstrativos, pode favorecer a compreensão do conceito de forma mais aprofundada (Zandonai et al., 2014; Gonçalves \& Marques, 2016).

A partir do tema eletroquímica, o Grupo 3 apresentou como uma de suas propostas experimentais a "pilha molhada", com o objetivo de analisar o funcionamento de uma célula eletroquímica e identificar suas relações cotidianas. Tal proposta se configura como uma nova abordagem para a demonstração de células eletroquímicas comumente desenvolvidas nos laboratórios de ensino (Cruz \& Filho, 2004; Guimarães \& Castro, 2018; Rodrigues et al., 2016; Barreto et al., 2017). Essa nova abordagem para a introdução do conceito de eletroquímica pôde contribuir para o processo didático da experimentação, como é possível observar a partir da comparação das respectivas Estrelas Verdes da Educação (EVE), apresentadas na Figura 7: 
Figura 7. EVE de um experimento de célula eletroquímica seguindo os princípios da EQV em comparação à proposta didática tradicional

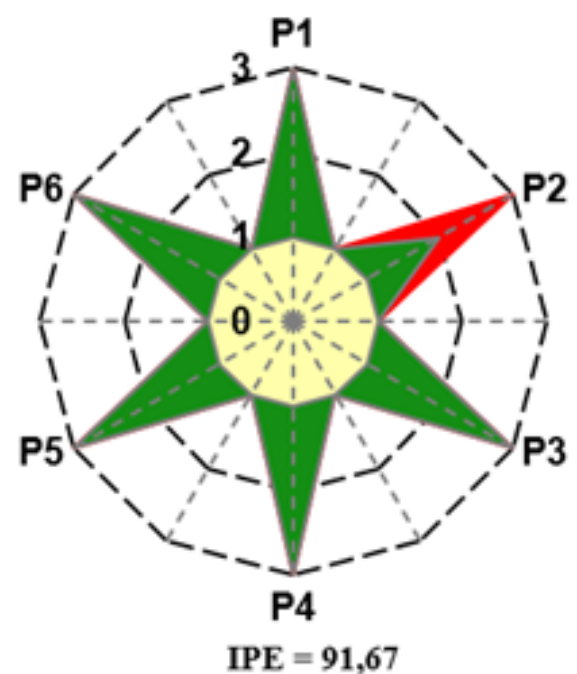

Células Eletroquímica EQV

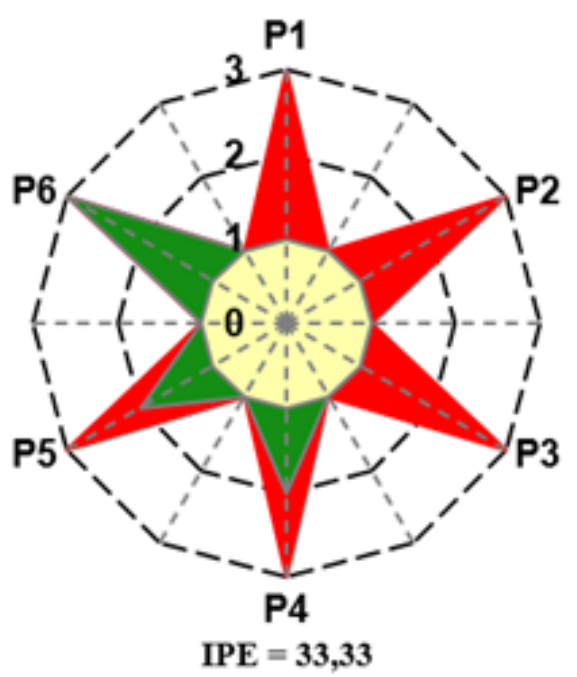

Células Eletroquímica Tradicional

Fonte: elaboração própria.

Ao analisar o Princípio P1, a proposta desenhada pelos licenciandos, além de utilizar materiais e reagentes presentes no cotidiano, apresenta a contextualização sobre o funcionamento, aplicação e descarte de células eletroquímicas, conferindo-lhe grau verde G3. Para as propostas experimentais tradicionais foi verificado grau verde G1, pois não se incluíram aspectos sociais, tecnológicos ou ambientais, restringindo-se a ou enfatizando apenas a abordagem dos conteúdos conceituais.

Em relação ao Princípio P2, a proposta didática EQV propiciou a tomada de decisão pelos estudantes quanto aos procedimentos e às conclusões da prática, sendo atribuído grau verde G2. Em contrapartida, as propostas já evidenciadas na literatura se apresentam como demonstrações simples e com conclusões já pré-definidas, o que não contribui no processo formativo do sujeito, sendo-lhes atribuído grau verde G1.

No experimento tradicional da célula eletroquímica não evidenciou-se nenhuma forma de minimização de resíduos, sendo atribuído ao Princípio P3 grau verde G1. No experimento da pilha molhada, a solução de íons metálicos de zinco produzida pelo funcionamento da pilha foi utilizada posteriormente na segunda prática experimental do grupo; portanto, o $\mathrm{Fe}(\mathrm{OH})_{2}$, resíduo dessa prática, foi totalmente utilizado como reagente da prática seguinte, ocasionando uma ressignificação do resíduo produzido e uma minimização dos impactos quanto ao seu descarte, o que lhe conferiu grau verde G3 para esse princípio.

Em relação ao Princípio P4, a proposta dos licenciandos apresentou grau verde G3, em função do uso de substâncias inócuas e por gerar produtos em baixa concentração, podendo ser utilizados como reagentes em outro experimento. Em geral, as propostas 
disponíveis na literatura utilizam substâncias de risco moderado para saúde e ambiente, como os sulfatos de cobre II $\left(\mathrm{CuSO}_{4}\right)$ e de $\mathrm{Zinco}\left(\mathrm{ZnSO}_{4}\right)$, sem qualquer alteração para minimizar suas concentrações.

Para o Princípio P5, a proposta dos licenciandos apresentou grau verde G3, por utilizar o sal de cozinha $(\mathrm{NaCl})$ como reagente. Já as propostas didáticas tradicionais empregam reagentes convencionais, mas que, por serem adquiridos no mercado nacional, reduzem o custo ambiental e econômico de seu transporte, o que resulta em grau verde G2. No que se refere ao Princípio P6, ambas as propostas se caracterizam com grau verde G3.

A estruturação e aplicação de propostas experimentais nas quais são inseridos problematização, incentivo à pesquisa e processos mais verdes possibilita aos professores em formação um entendimento mais adequado a respeito do papel da experimentação na construção do conhecimento formativo, além de propiciar compreensões coerentes acerca das práticas científicas.

Dessa forma, a inserção dos Princípios da Educação em Química Verde e o uso da métrica qualitativa Estrela Verde da Educação (EVE) como ferramenta para análise de experimentos didáticos permitiu identificar avanços no desenvolvimento de práticas experimentais em consonância com as demandas emergentes.

\section{Conclusões e Implicações}

Pautada na inserção dos Princípios da Educação em Química Verde como ferramenta avaliativa de experimentos didáticos, esta pesquisa traz algumas reflexões sobre a transição de práticas experimentais para além da aprendizagem instrumental e acrítica, mas que possibilitem ao estudante observar os desafios referentes a questões econômicas, sociais e ambientais mais amplas.

Assim como nos estudos de Zuin \& Marques (2015) e de Zuin e colaboradores (2020), nas propostas desenvolvidas não foi observada a inserção de situações-problema. Contudo, a maioria das propostas se estruturou em torno de um tema contextualizado, o que auxilia na compreensão e na aplicação da Química na sociedade (CNE/CES, 2001). Além disso, foi possível observar pela contextualização que os experimentos desenvolvidos possibilitaram a inserção da Química Verde nos processos educacionais.

Outra observação em relação às propostas desenvolvidas se refere à atenção dada pelos estudantes à escolha do processo, à redução de reagentes e produtos e à geração e uso de materiais perigosos. No entanto, ao escolher os regentes (Princípio 5), os grupos transitaram entre aqueles inócuos e os convencionais. Essa dificuldade em substituir reagentes convencionais por naturais ou comercialmente correspondentes - de uso doméstico - também é evidenciada por Kitchens et al., (2006) ao analisar concepções de estudantes de graduação em química sobre a integração da Química Verde em sua formação. Portanto, é possível observar a compreensão dos estudantes sobre a necessidade de se desenvolver um design simples com atenção aos perigos dos produtos químicos e preocupado com a redução de reagentes e dos resíduos gerados, rompendo com o reducionismo alertado por Zuin (2013) e desenvolvendo experimentos didáticos e seguros. 
De modo geral, as propostas experimentais discentes mostraram ter possibilitado uma reflexão e ressignificação da experimentação com base nos Princípios da Educação em Química Verde; portanto, é sinalizado que sua inserção na experimentação para a Educação Química deve apresentar, além de um design verde, a problematização e/ou contextualização como abordagens didática do experimento.

No entanto, também é sinalizado que ainda há um caminho a percorrer quando pensamos na Educação em Química Verde sob uma perspectiva para o Desenvolvimento Sustentável de forma transversal no currículo para a formação de professores e profissionais de química - principalmente quanto aos aspectos teóricos fundamentais, como abordagem do experimento, alfabetização científica e modelos de aprendizagem em Educação para o Desenvolvimento Sustentável, por exemplo, os quais norteiam a elaboração de propostas experimentais mais verdes na formação docente em química, além da necessidade de uma abordagem experimental que proporcione uma aprendizagem que envolva a Química em uma reflexão social, econômica e ambiental.

Por fim, diante dos dados e discussões, espera-se que os Princípios da Educação em Química Verde apresentados nesta pesquisa possam contribuir para o quadro metodológico acerca da implementação da Química Verde em atividades experimentais e de suas contribuições no processo formativo docente, favorecendo uma reflexão sobre a natureza da Educação Química contemporânea.

\section{Agradecimentos}

As autoras são gratas aos licenciandos participantes, às escolas parceiras e à equipe envolvida na condução da disciplina. Também agradecem à Caroindes Corrêa Gomes pelas contribuições e ao CNPq (311000/2014-2; 421096/2016-0; 310149/2017-7) e CAPES (88882.426363/2019-01) pelo fomento à pesquisa.

\section{Referências}

Almeida, P. C. A., \& Biajone, J. (2007). Saberes docentes e formação inicial de professores: implicações e desafios para as propostas de formação. Educação e Pesquisa, 33(2), 281295.

Anastas, P. T., \& Warner, J. (1998). Green chemistry: theory and practice. Oxford University Press.

Andraos, J., \& Sayed, M. (2007) On the Use of "Green" Metrics in the Undergraduate Organic Chemistry Lecture and Lab To Assess the Mass Efficiency of Organic Reactions. Journal of Chemical Education, 84(6), 1004. https://doi.org/10.1021/ed084p1004

Bazzo, W. A. A. (2002). A Pertinência de abordagens CTS na educação tecnológica. Revista Iberoamericana de Educación, (28).

Barreto, S. J., Batista, C. H., \& Cruz, M. C. P. (2017). Células Eletroquímicas, Cotidiano e Concepções dos Educandos. Química Nova na Escola, 39(1), 52-58. http://dx.doi. org/10.21577/0104-8899.20160060 
Capecchi, M. C. V. M. (2013). Problematização no ensino de ciências. In A. M. P. Carvalho (Org.), Ensino de ciências por investigação: condições para implementação em sala de aula (pp. 21-40). Cengage Learning.

Cárdenas, F. A. (2006). Dificultades de aprendizaje en Química: caracterización y búsqueda de alternativas para superarlas. Ciência \& Educação, 12(3), 333-346.

Creswell, J. (2010). Projeto de pesquisa: método qualitativo, quantitativo e misto. Artmed. Cruz, R., \& Filho, E. G. (2004). Experimentos em Química: microescala, materiais de baixo custo e do cotidiano. Livraria da Física.

Delizoicov, D., Angotti, J. A., \& Pernambuco, M. M. (2002). Ensino de ciências: fundamentos e métodos. Cortez.

Eilks, I., Sjostrom, J., \& Zuin, V. G. (2017). The responsibility of chemists for a better world: challenges and potentialities beyond the lab. Revista Brasileira de Ensino de Química, 12(1), 97-106.

Ferreira, G. A. L., Mól, G. S., \& Silva, R. R. (1997). Bafômetro: um modelo demonstrativo. Química Nova na Escola, 5, 32-33.

Flick, U. (2009). Introdução a pesquisa qualitativa (3a ed.). Artmed.

Furió, C., \& Furió, C. (2000). Dificultades conceptuales y epistemológicas en el aprendizaje de los procesos químicos. Educación Química, 11(3), 300-308. http://dx.doi. org/10.22201/fq.18708404e.2000.3.66442

Gonçalves, F. P., \& Marques, C. A. A. (2016). Experimentação na docência de formadores da área de ensino de Química. Química Nova na Escola, 38(1), 84-98. http://dx.doi. org/10.5935/0104-8899.20160013

Guimarães, L. P., \& Castro, D. L. (2018). A experimentação e a pilha de Daniell numa abordagem demonstrativa-investigativa. Ciências e Ideias, 9(3), 195-212.

Hjeresen, D. L., Schutt, D. L., \& Boese, J. M. (2000). Green Chemistry and Education. Journal of Chemical Education, 77(12), 1543. https://doi.org/10.1021/ed077p1543

Jiménez Valverde, G., Llobera Jiménez, R., \& Llitjós Viza, A. (2006). La atención a la diversidad en las prácticas de laboratorio de Química: los niveles de abertura. Enseñanza de las ciencias, 24(1), 59-70.

Kirchhoff, M. M. (2001). Topics in Green Chemistry. Journal of Chemical Education, 78(12), 1577. https://doi.org/10.1021/ed078p1577

Kitchens, C., Charney, R., Naistat, D., Farrugia, J., Clarens, A., O'Neil, A., Lisowski, C., \& Braun, B. (2006). Completing Our Education. Green Chemistry in the Curriculum. Journal of Chemical Education, 83(8), 1126-1129. https://doi.org/10.1021/ed083p1126 
Lenardão, E. J., Freitag, R. A., Dabdoub, M. J., Batista, A. C. F., \& Silveira, C. C. (2003). 'Green chemistry': Os 12 princípios da Química Verde e sua inserção nas atividades de ensino e pesquisa. Química Nova, 26(1), 123. https://doi.org/10.1590/S010040422003000100020

Machado, A. A. S. C. (2011). Da génese ao ensino da Química verde. Química Nova, 34(3), 535-543. https://doi.org/10.1590/S0100-40422011000300029

Marques, C. A., \& Machado, A. A. S. C. (2018). Una visión sobre propuestas de enseñanza de la Química Verde. Revista Electrónica de Enseñanza de las Ciencias, 17(1), 19-43.

Montes, I., Sanabria, D., Garcia, M., Castro, J., \& Fajardo, J. A. (2006). Greener approach to aspirin synthesis using microwave irradiation. Journal of Chemical Education, 83(4), 628. https://doi.org/10.1021/ed083p628

Morales Galicia, M. L., Martínez, J. O., Reyes-Sánchez, L. B., Hernández, O. M., Razo, G. A. A., Valdivia, A. O., \& Ruvalcaba, R. M. (2011). ¿Qué tan verde es un experimento? Educación química, 22(3), 240-248.

Parecer CNE/CES 1.303/2001 de 06 de novembro de 2001 (2001). Diretrizes Curriculares Nacionais para os Cursos de Química. Conselho Nacional de Educação/Ministério da Educação. http://portal.mec.gov.br/sesu/arquivos/pdf/130301Quimica.pdf

Pinto, A. C., Zucco, C., De Andrade, J. B., \& Vieira, P. C. (2009). Recursos humanos para novos cenários. Química Nova, 32(3), 567-570. https://doi.org/10.1590/S010040422009000300002

Pozo, J. I., \& Crespo, M. A. G. (2009). A aprendizagem e o ensino de ciências: do conhecimento cotidiano ao conhecimento científico. Artmed.

Ribeiro, M. G. T. C., \& Machado, A. A. S. C. (2013). Holistic Metrics for Assessment of the Greenness of Chemical Reactions in the Context of Chemical Education. Journal of Chemical Education, 90(4), 432-439. https://doi.org/10.1021/ed300232w

Ribeiro, M. G. T. C., Costa, D. A., \& Machado, A. A. S. C. (2010a). Uma métrica gráfica para avaliação holística da verdura de reacções laboratoriais - 'Estrela verde'. Química Nova, 33(3), 759-764. https://doi.org/10.1590/S0100-40422010000300050

Ribeiro, M. G. T. C., Costa, D. A., \& Machado, A. A. S. C. (2010b). "Green star": a holistic greenchemistrymetricfor evaluation ofteachinglaboratoryexperiments. Green Chemistry Letters and Reviews, 3(2), 149-159. https://doi.org/10.1080/17518251003623376

Rodrigues, F. I. L., Fernandes Filho, F. E., Da Silva, J. E., Da Silva, M. A., De Lima Neto, A. L., Nonato, E. C., Da Silva, M. N. F., Pacífico, S. N., Sousa Neo, V. O., \& Santiago, L. F. (07-11 de novembro, 2016). Práticas experimentais de eletroquímica no ensino de química: aplicadas com alunos do ensino médio de uma escola profissional do município de Tauá. 56 Congresso Brasileiro de Química, Belém, Pará. 
Rubinger, M. M. M., \& Braathen, P. C. (2012). Ação e reação: Ideias para aulas especiais de Química. RHJ.

Santos, A. P., Santos, A. S., Santos, I. A. P., Santos, M. C., Silva, J. S., Silva, M. B., \& Junior, A. C. (2014). O teste do bafômetro: contextualização através de experimento na sala de aula. 54 Congresso Brasileiro de Química, Natal, Rio Grande do Norte.

Santos, W. L. P., \& Mortimer, E. F. (2002). Uma análise de pressupostos teóricos da abordagem C-T-S (Ciência-Tecnologia-Sociedade) no contexto da educação brasileira. Ensaio: Pesquisa em Educação em Ciências, 2(2), 110-132. https://doi.org/10.1590/198321172000020202

Saqueto, K. C. (2015). Química verde no ensino superior de Química: estudo de caso sobre as práticas vigentes em uma IES paulista (Tese de Doutorado, São Carlos, São Paulo). Repositório Institucional - UFSCar. https://repositorio.ufscar.br/handle/ufscar/6345

Silva, R. R., Machado, P. F. L., \& Tunes, E. (2010). Experimentar sem medo de errar. In W. L. Santos, O. A. Maldaner (Org.), Ensino de Química em foco (pp. 231-261). Unijuí.

Vargas-Rodrígueza, Y. M., Valdivia, A. O., Vargas, S. L., Escamilla, A. H., Ruvalcaba, R. M., \& Rodríguez, G. I. V. (2016). El diagrama de flujo como semáforo de seguridad ecológica de los experimentos de laboratório. Educación Química, 27(1), 30-36. https:// doi.org/10.1016/j.eq.2015.04.013

Zandonai, D. P., Saqueto, K. C., Abreu, S. C. S. R., Lopes, A. P., Zuin, V. G. (2014). Química Verde e Formação de Profissionais do Campo da Química: Relato de uma Experiência Didática para Além do Laboratório de Ensino. Revista Virtual de Química, 6, 73-84. http://dx.doi.org/10.5935/1984-6835.20140007

Zuin, V. G., \& Marques, C. A. (2015). Green Chemistry in Brazil: Contemporary Tendencies and Challenges and its Reflections on High School Level. In V. G. Zuin \& L. Mammino (Org). Worldwide Trends in Green Chemistry (pp. 27-44). RSC.

Zuin, V. G., \& Pacca, J. L. A. (2012). Formación docente en química y ambientación curricular: estudio de caso en una Instituición de Enseñanza Brasileña. Enseñanza de las Ciencias, 31, 77-91.

Zuin, V. G. (2011). A inserção da dimensão ambiental na formação de professores de Química. Átomo.

Zuin, V. G. (2013). A dimensão ambiental e a Química Verde na formação inicial de professores de Química: reflexões a partir de um estudo de caso. Revista Brasileira de Ensino de Química, 8, 70-82.

Zuin, V. G., Segatto, M. L., Zandonai, D. P., Grosseli, G. M., Stahl, A., Zanotti, K., \& Andrade, R. S. (2019). Integrating Green and Sustainable Chemistry into Undergraduate Teaching Laboratories: Closing and Assessing the Loop on the Basis of a Citrus Biorefinery Approach for the Biocircular Economy in Brazil. Journal of Chemical Education, 96(12), 2975-2983. https://doi.org/10.1021/acs.jchemed.9b00286 
Zuin, V. G., Stahl, A., Zanotti, K., \& Segatto, M. L. (2020) Green and sustainable chemistry in Latin America: which type of research is going on? And for what?. Current Opinion In Green And Sustainable Chemistry, 25, 100379-100384.

Zuin, V. G., \& Zuin, A. A. S. (2017). O laboratório de Química como lócus de experiências formativas. Ensaio: Pesquisa em Educação em Ciências, 19, 1-16. http:// dx.doi.org/10.1590/1983-21172017190108

\author{
Rosivânia da Silva Andrade \\ Universidade Federal de São Carlos \\ São Carlos, São Paulo, Brasil \\ rosivaniandrade@gmail.com \\ Vânia Gomes Zuin \\ Universidade Federal de São Carlos \\ Leuphana University Lüneburg \\ University of York \\ São Carlos, São Paulo, Brasil \\ vaniaz@ufscar.br \\ vania.zuin@leuphana.de
}

Editora Responsável

Stefannie Ibraim

Manifestação de Atenção às Boas Práticas Científicas e de Isenção de Interesse

Os autores declaram ter cuidado de aspectos éticos ao longo do desenvolvimento da pesquisa e não ter qualquer interesse concorrente ou relações pessoais que possam ter influenciado o trabalho relatado no texto. 